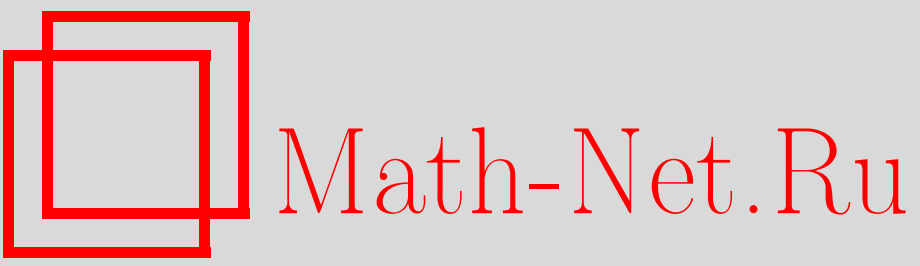

А. А. Голдаева, Некоторые неравенства для броуновского движения со сносом, УМH, 2002, том 57, выпуск 6, 173-174

DOI: https://doi.org/10.4213/rm578

Использование Общероссийского математического портала Math-Net.Ru подразумевает, что вы прочитали и согласны с пользовательским соглашением

http://www.mathnet.ru/rus/agreement

Параметры загрузки:

IP : 3.82 .47 .9

26 апреля 2023 г., 14:41:26 


\title{
НЕКОТОРЫЕ НЕРАВЕНСТВА ДЛЯ БРОУНОВСКОГО ДВИЖЕНИЯ СО СНОСОМ
}

\author{
А. А. ГОЛДАЕВА
}

Целю данной работы является установление следующего неравенства.

Tеорема 1. Пусть $B^{\mu}(t)=B(t)+\mu t-$ броуновское движение со сносом, $f:[0,1] \rightarrow$ $\{+1,-1\}-$ измеримая детерминированная функиия. Тогда для любого $t \geqslant 0$ имеем:

$$
\begin{aligned}
\mathrm{P}\left(\max _{0 \leqslant s \leqslant 1}\left|B^{\mu}(s)\right| \geqslant t\right) & \leqslant \mathrm{P}\left(\max _{0 \leqslant s \leqslant 1}\left|\int_{0}^{s} f(u) d B^{\mu}(u)\right| \geqslant t\right) \\
& \leqslant \mathrm{P}\left(\max _{0 \leqslant s \leqslant 1}\left|B^{\mu}(s)\right| \geqslant \frac{t}{2}\right) .
\end{aligned}
$$

Сначала докажем следующее утверждение.

TeOpema 2.

$$
\begin{aligned}
\mathrm{P}\left(\max _{0 \leqslant s \leqslant 1}|B(s)| \geqslant t\right) & \leqslant \mathrm{P}\left(\max _{0 \leqslant s \leqslant 1}\left|\int_{0}^{s} f(u) d B(u)\right| \geqslant t\right) \\
& \leqslant \mathrm{P}\left(\max _{0 \leqslant s \leqslant 1}|B(s)| \geqslant \frac{t}{2}\right),
\end{aligned}
$$

где $B(t)$ - броуновское движение.

Шаг 1. Предположим, что функция $f$ имеет вид

$$
f(t)=\sum_{k=0}^{N} \varepsilon_{k} \mathrm{I}_{\left[a_{k}, a_{k+1}\right)}(t), \quad 0 \leqslant t \leqslant 1
$$

где $\varepsilon_{k}= \pm 1,0=a_{0}<\cdots<a_{N}=1, N<\infty$. Воспользуемся следующей теоремой из [1]:

Теорема 3. Пусть $\theta=\left(\theta_{1}, \ldots, \theta_{2 n}\right)$ u $x=\left(x_{1}, \ldots, x_{2 n}\right)$ - последовательности знаков. Возьмем в качестве $\mathrm{P}$ равномерное распределение на $\{\pi\}$, множестве всех перестановок чисел $\{1, \ldots, 2 n\}$. Тогда

$$
\mathrm{P}\left(\left|x_{\pi}\right| \geqslant t\right) \leqslant \mathrm{P}\left(\left|\theta \cdot x_{\pi}\right| \geqslant t\right) \leqslant \mathrm{P}\left(\left|x_{\pi}\right| \geqslant \frac{t}{2}\right) \quad \forall \theta \in \Theta, \quad \forall t \geqslant 0,
$$

əде $x_{\pi}=\left(x_{\pi(1)}, \ldots, x_{\pi(2 n)}\right), \theta \cdot x_{\pi}=\left(\theta_{1} x_{\pi(1)}, \ldots, \theta_{2 n} x_{\pi(2 n)}\right) u$

$$
\left|x_{\pi}\right|=\max _{1 \leqslant k \leqslant 2 n}\left\|\sum_{i=1}^{k} x_{\pi(i)}\right\|
$$

Определим для любого $n>0, x \in\left\{x_{\pi}\right\}$ следующий процесс: $Z_{n}(t)=\frac{\sum_{i=1}^{[2 n t]} x_{i}}{\sqrt{2 n}}, 0 \leqslant t \leqslant 1$, где $[y]$ - целая часть $y$. Положим $Y_{n}(t)=\int_{0}^{t} f(u) d Z_{n}(u)$ и $Y(t)=\int_{0}^{t} f(u) d B(u), 0 \leqslant t \leqslant 1$. Очевидно,

$$
\begin{aligned}
& \max _{0 \leqslant s \leqslant 1}\left|Y_{n}(s)\right|=\max _{1 \leqslant k \leqslant 2 n}\left|\frac{\sum_{i=1}^{k} f\left(\frac{i}{2 n}\right) x_{i}}{\sqrt{2 n}}\right|, \\
& \max _{0 \leqslant s \leqslant 1}\left|Z_{n}(s)\right|=\max _{1 \leqslant k \leqslant 2 n}\left|\frac{\sum_{i=1}^{k} x_{i}}{\sqrt{2 n}}\right|=\frac{\left|x_{i}\right|}{\sqrt{2 n}} .
\end{aligned}
$$


Из теоремы 3 следует, что $\mathrm{P}\left(\max _{0 \leqslant s \leqslant 1}\left|Y_{n}(s)\right| \geqslant t\right) \geqslant \mathrm{P}\left(\max _{0 \leqslant s \leqslant 1}\left|Z_{n}(s)\right| \geqslant t\right)$. Известно, что $Z_{n} \Rightarrow B$, где $\Rightarrow$ означает сходимость по распределению (см. [2]). Так как отображение $X(\cdot) \rightarrow \int_{0}^{\cdot} f(u) d X(u)$ непрерывно на пространстве ограниченных функций на $[0,1]$, то $Y_{n} \Rightarrow Y$. Таким образом, $\mathrm{P}\left(\max _{0 \leqslant s \leqslant 1}|Y(s)| \geqslant t\right) \geqslant \mathrm{P}\left(\max _{0 \leqslant s \leqslant 1}|B(s)| \geqslant t\right)$.

Шаг 2. Общий случай. Измеримую детерминированную функцию $f:[0,1] \rightarrow\{+1,-1\}$ аппроксимируем последовательностью функций типа $(3): \int_{0}^{1}\left(f_{n}(u)-f(u)\right)^{2} d u \rightarrow 0$. Испольуя неравенство Дуба:

получаем

$$
\mathrm{E}\left(\max _{0 \leqslant s \leqslant 1}\left|\int_{0}^{s}\left(f_{n}(u)-f(u)\right) d B(u)\right|\right)^{2} \leqslant 4 \int_{0}^{1}\left(f_{n}(u)-f(u)\right)^{2} d u
$$

$$
\max _{0 \leqslant s \leqslant 1}\left|\int_{0}^{s}\left(f_{n}(u)-f(u)\right) d B(u)\right| \stackrel{\mathrm{P}}{\longrightarrow} 0 .
$$

Таким образом, с помощью шага 1 и (6) первое неравенство в (2) доказано. Второе неравенство в (2) доказывается аналогично.

Для доказательства (1) применим теорему Гирсанова. Пусть

$$
F(x)=\max _{0 \leqslant s \leqslant 1}|X(s)|, \quad G(X)=\max _{0 \leqslant s \leqslant 1}\left|\int_{0}^{s} f(u) d X(u)\right|,
$$

где $X$ - точка в пространстве непрерывных функций. Тогда

$$
\begin{aligned}
\mathrm{P}^{\mu}(F(X) \geqslant t) & =\mathrm{E}^{\mu} \mathrm{I}(F(X) \geqslant t)=\mathrm{E}^{0} \frac{d \mathrm{P}_{1}^{\mu}}{d \mathrm{P}_{1}^{0}} \mathrm{I}(F(X) \geqslant 1)=\mathrm{E}^{0} e^{\mu B_{1}-\frac{\mu^{2}}{2}} \mathrm{I}(F(B) \geqslant t) \\
& =\mathrm{E}^{0} \mathrm{E}\left(e^{\mu B_{1}-\frac{\mu^{2}}{2}} \mathrm{I}(F(B) \geqslant t) \mid B_{1}\right)=\mathrm{E}^{0} e^{\mu B_{1}-\frac{\mu^{2}}{2}} \mathrm{E}\left(\mathrm{I}(F(B) \geqslant t) \mid B_{1}\right),
\end{aligned}
$$

где $\mathrm{P}^{0}$ и $\mathrm{P}^{\mu}$ - меры, отвечающие броуновскому движению и броуновскому движению со сносом соответственно. Аналогично

$$
\mathrm{P}^{\mu}(G(X) \geqslant t)=\mathrm{E}^{0} e^{\mu B_{1}-\frac{\mu^{2}}{2}} \mathrm{E}\left(\mathrm{I}(G(B) \geqslant t) \mid B_{1}\right)
$$

и

$$
\mathrm{P}^{\mu}(F(X) \geqslant t / 2)=\mathrm{E}^{0} e^{\mu B_{1}-\frac{\mu^{2}}{2}} \mathrm{E}\left((F(B) \geqslant t / 2) \mid B_{1}\right) .
$$

Из теоремы 2 следует, что

$$
\mathrm{E}\left(\mathrm{I}(F(B) \geqslant t) \mid B_{1}\right) \leqslant \mathrm{E}\left(\mathrm{I}(G(B) \geqslant t) \mid B_{1}\right) \leqslant \mathrm{E}\left(\mathrm{I}(F(B) \geqslant t / 2) \mid B_{1}\right) .
$$

Таким образом, приходим к следующему резултату:

$$
\mathrm{P}^{\mu}(F(X) \geqslant t) \leqslant \mathrm{P}^{\mu}(G(X) \geqslant t) \leqslant \mathrm{P}^{\mu}(F(X) \geqslant t / 2) .
$$

Перепишем (7), используя обозначения для $F$ и $G$ :

$$
\mathrm{P}\left(\max _{0 \leqslant s \leqslant 1}\left|B^{\mu}(s)\right| \geqslant t\right) \leqslant \mathrm{P}\left(\max _{0 \leqslant s \leqslant 1}\left|\int_{0}^{s} f(u) d B^{\mu}(u)\right| \geqslant t\right) \leqslant \mathrm{P}\left(\max _{0 \leqslant s \leqslant 1}\left|B^{\mu}(s)\right| \geqslant t / 2\right) .
$$

Получаем требуемое неравенство.

\section{СПИСОК ЛИТЕРАТУРЫ}

[1] S. Levental // Statist. Probab. Lett. 2000. V. 46. № 3. P. 271-276. [2] J. Hajek, Z. Sidak. Theory of Rank Tests. New York: Academic Press, 1967. 